\title{
Tipo de juez y estimación de los casos de despido: un análisis de los Juzgados de lo Social en España
}

\author{
Ángel L. Martín Román*, Alfonso Moral de Blas y Marta Martínez Matute \\ Facultad de Ciencias Sociales, Jurídicas y de la Comunicación, Universidad de Valladolid, Segovia, España
}

Recibido el 8 de enero de 2013; aceptado el 9 de abril de 2013

Disponible en Internet el 18 de septiembre de 2013

\section{CÓDIGOS JEL \\ $\mathrm{J} 65$; \\ $\mathrm{K} 31$; \\ K41}

\section{PALABRAS CLAVE}

Despidos;

Decisiones judiciales;

Juzgados de lo Social

\section{JEL CLASSIFICATION $\mathrm{J} 65$; \\ $\mathrm{K} 31$; \\ K41}

\section{KEYWORDS}

Dismissals;

Judges' decisions;

Labour courts

\begin{abstract}
Resumen El objetivo de este artículo es profundizar en la relación existente entre las decisiones judiciales de lo social en España en los casos de despido y los costes asociados a la extinción de los contratos laborales. Primero justificamos que los cambios en la probabilidad de que un juez falle a favor de un trabajador afectan a los costes de despido del empleador. En segundo lugar, examinamos cómo difiere dicha probabilidad cuando el juez es titular o sustituto. Se observa una mayor dispersión en la resolución de los casos de los jueces sustitutos, y un significativo repunte en los años de crisis económica de los fallos judiciales a favor del trabajador al considerar los jueces titulares, siendo este más débil en los casos de los sustitutos.

(c) 2013 Asociación Cuadernos de Economía. Publicado por Elsevier España, S.L. Todos los derechos reservados.
\end{abstract}

Type of judge and decisions in dismissal cases: An analysis of Labour Courts in Spain

Abstract The aim of this paper is to examine in detail the relationship between the decisions of the Spanish labour courts regarding dismissal, and the costs associated with the termination of employment contracts. We explain the changes in the probability that a judge ruling in favour of a worker affects the effective firing costs that the employer faces. We also examine how this probability differs depending on whether the judge is in charge of the court or is a substitute. There is a greater dispersion in the resolution of cases when there are substitute judges, with a significant rise, during the economic crisis, of court decisions in favour of the workers with permanent judges, which is less so with substitute judges.

(C) 2013 Asociación Cuadernos de Economía. Published by Elsevier España, S.L. All rights reserved.

\footnotetext{
* Autor para correspondencia.

Correo electrónico: angellm@eco.uva.es (Á.L. Martín Román).
}

\section{Introducción}

La pregunta a la que pretende dar respuesta este trabajo es si los jueces titulares y sustitutos de la jurisdicción laboral se comportan de igual manera en los asuntos relativos a despidos, lo que pensamos que tiene un efecto claro sobre 
el coste que afronta un empleador que pretende extinguir una relación contractual laboral.

Dar respuesta a esta pregunta tiene interés por varios motivos. El primero de ellos, y quizás el más inmediato por motivos de coyuntura política, es que actualmente se está llevando a cabo en España una reforma judicial, y uno de sus puntos es precisamente la reducción del peso que tienen los jueces sustitutos dentro del sistema jurídico ${ }^{1}$. Una mejor comprensión de las diferencias existentes en la forma de desarrollar su trabajo por parte de ambos colectivos puede arrojar una luz muy valiosa para «iluminar» el debate político. Aunque nuestro artículo se circunscribe a la jurisdicción de lo Social, es una importante contribución al marco más general del debate.

Un segundo motivo, aunque íntimamente ligado con el primero, es que el porcentaje de asuntos resueltos por los jueces sustitutos en materia laboral no es ni mucho menos una magnitud despreciable. Así, por ejemplo, durante el periodo de tiempo que vamos a considerar en esta investigación (desde 2004 hasta 2011) el porcentaje de asuntos de despido resueltos por los jueces sustitutos ha superado siempre el $10 \%$. Más aún, durante los años de crisis económica, además de aumentar el número de casos de despidos individuales, también lo ha hecho el porcentaje de estos que son resueltos por jueces sustitutos, llegando en el año 2011 a suponer el $19,45 \%$ del total. Con estas cifras, parece evidente que estudiar un fenómeno como las diferencias en el comportamiento de jueces titulares y sustitutos tiene trascendencia socioeconómica.

Finalmente, un último motivo es que en el ámbito temporal que consideramos en la presente investigación se ha producido un fuerte incremento del número de asuntos de despido que llegan a la vía judicial. Según las estadísticas del Consejo General del Poder Judicial (CGPJ), en el año 2004 los jueces de lo Social resolvieron 29.707 asuntos de despido, mientras que en 2011 esta cifra ascendió a 44.665. El resultado final es un fuerte incremento del 50,4\%. En otras palabras, durante los años de bonanza económica el recurso a la vía judicial fue mucho menor que en los años de crisis económica. Como se pone de manifiesto en el trabajo de Frick et al. (2012), las condiciones económicas (en especial la tasa de desempleo) son un factor determinante del recurso a los tribunales por parte de los trabajadores. Dado ese incremento en el volumen de casos que llegan a las salas de lo Social, parece interesante investigar si eso ha condicionado el sentido de los fallos emitidos por los jueces, y especialmente si se han producido diferencias significativas en el comportamiento de los titulares del juzgado y de los sustitutos.

Este artículo intenta ubicarse entre los campos de investigación de la Economía Laboral (EL) y el Análisis Económico del Derecho (AED). Por una parte, está muy relacionado con la literatura académica sobre costes de despido propia de la EL y que tiene como uno de sus primeros y principales hitos el trabajo de Lazear (1990). A la vez, también estaría muy ligado a otra literatura, más propia del AED,

\footnotetext{
${ }^{1}$ Una información periodística relacionada con dicha cuestión puede encontrarse en: http://www.elderecho.com/actualidad/ Congreso-Senado-sustitutos-permisos-magistrados_0_478875283. html
}

que examina los determinantes de las decisiones judiciales. Un ejemplo de esta bibliografía académica es el trabajo de Cooter y Rubinfeld (1989). En una sección posterior se presentará un sencillo marco analítico en el que se mostrará que hay una fuerte conexión entre las decisiones judiciales en materia de despido y los costes de extinción de una relación laboral.

Finalmente, como breve adelanto de los principales resultados obtenidos en esta investigación, habría que decir que a pesar de que parecen no apreciarse grandes diferencias al analizar la probabilidad media de fallo judicial a favor del trabajador cuando se desglosa por tipo de juez y en el periodo 2004-2011, un análisis más pormenorizado pone de manifiesto 2 resultados relevantes. El primero es que se observa una mayor dispersión en la resolución de los casos cuando se trata de jueces sustitutos. El segundo es que se produce una significativa asimetría cíclica. En los años de crisis económica se ha producido un significativo repunte de los fallos judiciales a favor del trabajador cuando se consideran los jueces titulares y un repunte muy débil o no estadísticamente significativo cuando se tienen en cuenta los jueces sustitutos.

El resto del trabajo se estructura de la siguiente manera. En la sección 2 se describe el marco jurídico de los conflictos laborales en España. En la sección 3 se revisa la literatura académica sobre el tema. Se incluye una cuarta sección en la que se presenta un simple marco teórico que pretende ilustrar la conexión que existe entre las decisiones judiciales y los costes de despido. El quinto apartado explica y comenta la base de datos utilizada en esta investigación. Los apartados 6 y 7 son de carácter empírico, y en ellos se obtienen los principales resultados del trabajo. Se finaliza el artículo con la habitual sección de conclusiones.

\section{El marco jurídico de los conflictos laborales en España}

Cuando un trabajador es despedido, puede optar por reclamar dicho procedimiento a través del órgano judicial correspondiente. En la legislación española existe la opción previa y voluntaria de resolver dicho conflicto acudiendo, tanto él como el empresario, a una Unidad de Mediación, Arbitraje y Conciliación (Unidades MAC). Del mismo modo que se establece en la legislación internacional, este es un mecanismo para resolver disputas individuales o colectivas en el ámbito de las relaciones laborales. En España, esta unidad constituye un órgano extrajudicial de resolución de conflictos a disposición de cada autoridad regional. Si a través de las Unidades MAC trabajador y empresario no llegan a un acuerdo, el trabajador puede continuar su proceso de reclamación acudiendo a un juzgado de lo Social.

En la estructura judicial española existen distintos niveles jerárquicos. En primera instancia, el caso llega al Juzgado de lo Social correspondiente. El Juzgado de lo Social está situado en la ciudad donde el trabajador tiene su empleo. Si la decisión del juez es recurrida por una de las partes, el asunto podría llegar a la Sala de lo Social del Tribunal Superior de Justicia de la comunidad autónoma correspondiente. Finalmente, esta decisión se podría apelar ante la Sala de lo Social del Tribunal Supremo. 


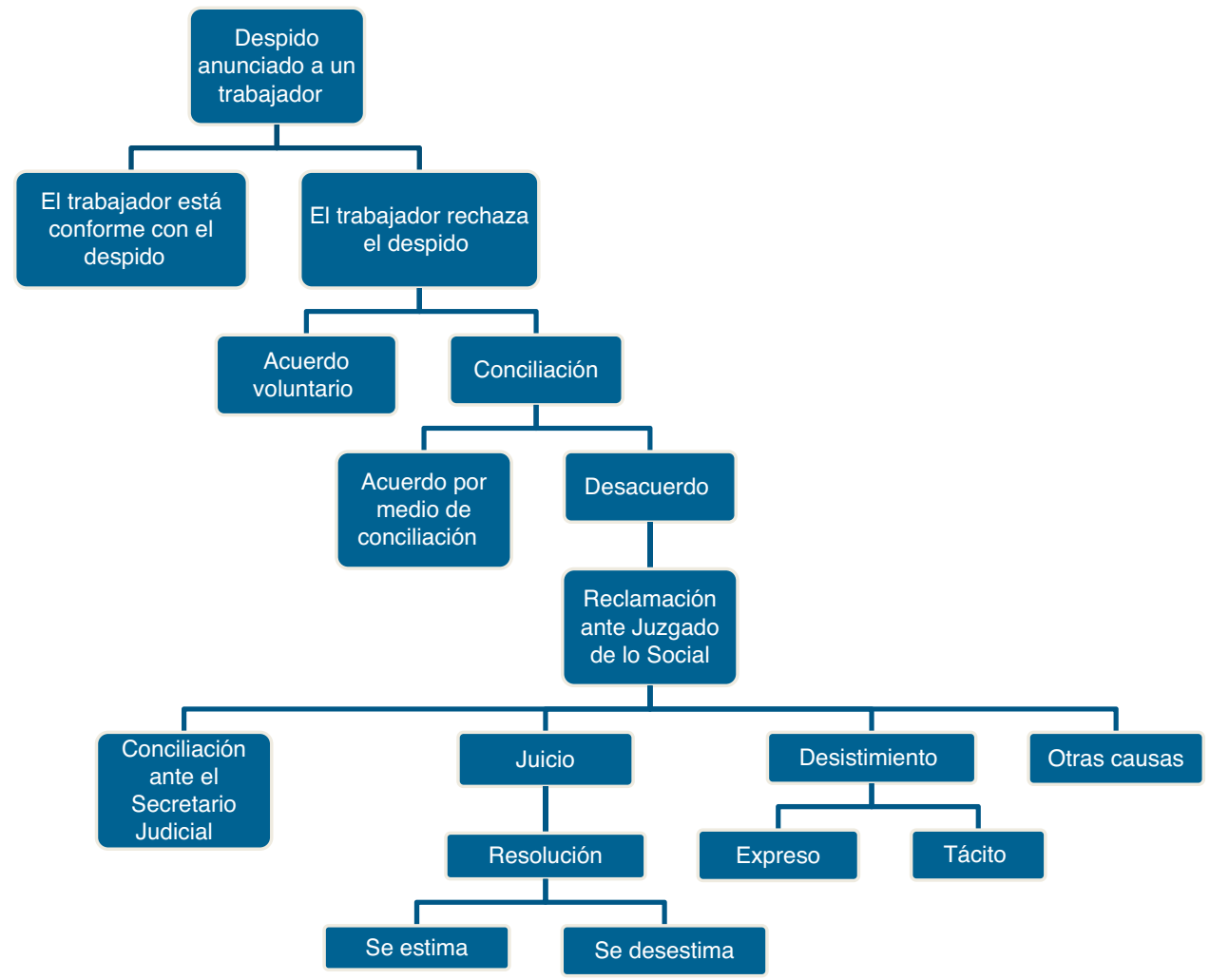

Figura 1 Procedimiento de reclamación de un despido en el sistema judicial español. Fuente: Elaboración propia a partir de Frick et al. (2012).

El procedimiento habitual que sigue un asunto que ingresa en un Juzgado de lo Social es, en términos generales, el que se presenta en la figura 1.

En primer lugar, cuando la reclamación de un despido alcanza el Juzgado de lo Social, el Secretario Judicial de dicho juzgado reúne a las partes en audiencia pública y trata de llevar a cabo una conciliación previa entre ellas. Si no se alcanza la avenencia, las partes irían a juicio, y el magistrado correspondiente tendría que sentenciar estimando o desestimando la demanda. Aparte de estas circunstancias, puede suceder que la parte promotora desista (tácita o expresamente) del conflicto durante el procedimiento o que concurran otras causas que impidan al juez tomar una decisión (tales como la existencia de fallos de forma, falta de la documentación requerida, la inhibición del juzgado en que se ha presentado la demanda en favor de otro juzgado, que este no tenga competencia, o que el asunto se archive por alguna circunstancia). Por lo tanto, no todos los asuntos que entran en los juzgados de lo Social llegan a ser resueltos por un juez.

Cabe mencionar que si existen 2 o más juzgados en un mismo orden jurisdiccional, los asuntos se distribuyen entre ellos conforme a unas reglas de reparto ${ }^{2}$ que, ante todo, garantizan la aleatoriedad en la asignación de los jueces

\footnotetext{
2 De acuerdo con el artículo 167 de la Ley Orgánica del Poder Judicial, las reglas de reparto han de ser aprobadas por la Sala de Gobierno del Tribunal Superior de Justicia, a propuesta de la Junta de Jueces del respectivo orden jurisdiccional.
}

responsables. Desde la presentación de la demanda, denuncia, querella o cualquier pretensión principal que se ejercite ante los órganos judiciales, los servicios del Decanato, o el órgano judicial, en su caso, atribuyen al procedimiento un número de identificación. En un plazo de 2 días se lleva a cabo un reparto del asunto a la Oficina Judicial correspondiente. De acuerdo con el artículo 167 de la Ley Orgánica del Poder Judicial (LOPJ), con el artículo 25 del Reglamento $1 / 2005$ de los aspectos accesorios de las actuaciones judiciales, y de los artículos 68, 69 y 70 de la Ley de Enjuiciamiento Civil, el reparto de los casos se efectúa de forma informática, asegurándose así un reparto igualitario entre todos los juzgados. Este reparto se realiza introduciendo los datos de las demandas en un ordenador (prestando especial atención al tipo de procedimiento y a la materia tratada), y el ordenador asigna automáticamente un juzgado conforme a un algoritmo de reparto que se basa en un sorteo secuencial y aleatorio. Aunque las reglas de reparto son públicas y el resultado del reparto podrá ser conocido por los interesados mediante solicitud de comprobación del asiento dirigido al Juzgado Decano, una vez iniciado el procedimiento no es posible modificar la asignación de un juez.

Además de las reclamaciones sobre despidos, en las cuales se centra esta exposición, existen distintos tipos de asuntos que entran en un Juzgado de lo Social. El CGPJ establece la siguiente clasificación, distinguiendo principalmente entre:

- Asuntos sobre conflictos colectivos: recogen los procedimientos que afectan a intereses de un grupo genérico de 
trabajadores considerado en su conjunto o en abstracto. Tratan generalmente sobre la aplicación o interpretación de una norma, de un convenio colectivo o de una decisión o práctica de empresa. También a través de este procedimiento se tramita la impugnación de convenios colectivos. Pueden ser promovidos por sindicatos, asociaciones empresariales, empresarios y órganos de representación legal o sindical de los trabajadores en caso de ser conflictos de empresa o centro de trabajo.

- Asuntos sobre cantidades: recogen los conflictos relacionados con sueldos y salarios, complementos salariales, pagas extraordinarias, etc.

- Asuntos sobre Seguridad Social: relacionados con reclamaciones de materias tal que afiliación, cotización, prestaciones, accidentes de trabajo y enfermedades profesionales, etc.

- Asuntos de otra índole: como por ejemplo los relativos a vacaciones, condiciones de trabajo particulares, y también aquellos en los que la parte demandante pueda ser la Inspección de Trabajo.

Es importante remarcar que en los conflictos en materia de despidos, la reclamación se realiza siempre por parte del trabajador. Esto nos permite identificar los casos de despido que son estimados en un juzgado de lo Social como asuntos favorables al trabajador, y por el contrario, los casos desestimados resultan desfavorables con respecto al trabajador. Esto no siempre sucede en otro tipo de asuntos, ya que, por ejemplo, en el caso de reclamaciones relacionadas con la Seguridad Social, la propia autoridad pública o la Inspección de Trabajo podrían personarse como reclamante. También en los conflictos colectivos los sindicatos y las asociaciones empresariales, por ejemplo, pueden actuar como tales.

Dado que el objetivo último de este trabajo radica en el análisis de los distintos tipos de jueces de lo Social, vamos a dedicar también unas líneas a describir en qué se basan estas diferencias. En primer lugar, la clasificación que establece el CGPJ es la que distingue entre los siguientes tipos de magistrados: titular, sustituto o suplente, refuerzo, titular de otro juzgado y otros. Las más importantes, y en las que hemos centrado este artículo, son las 2 primeras. Por tanto, podemos caracterizar estas 2 clases de jueces del siguiente modo:

- Juez titular: son los magistrados pertenecientes a la Carrera Judicial que han superado una oposición libre o bien un concurso entre juristas de reconocida competencia con más de 10 años de experiencia profesional, además de un curso teórico y práctico realizado en la Escuela Judicial, tal y como se menciona en los artículos 301.3, 301.5, 311.1, 311.2 de la LOPJ.

- Juez sustituto o magistrado suplente ${ }^{3}$ : ejercen funciones jurisdiccionales sin pertenecer a la Carrera Judicial, sin carácter de profesionalidad y con inamovilidad temporal, tal como dispone el artículo 298.2 de la LOPJ, aunque actúan como miembros del juzgado correspondiente con los mismos derechos y deberes que sus titulares, de conformidad con lo previsto en los artículos 200.3 y 212.2

\footnotetext{
${ }^{3}$ A lo largo del trabajo hablaremos indistintamente de jueces sustitutos o jueces suplentes.
}

de la citada Ley. El CGPJ es el encargado de proponer la adscripción de dichos jueces sustitutos a un determinado tribunal o juzgado y de efectuar su nombramiento para el siguiente año judicial, a tenor de lo mencionado en el Reglamento 2/2011 de la Carrera Judicial. Estos conforman unas listas gestionadas por los Tribunales Superiores de Justicia, son solamente remunerados por los días que desempeñen dichas funciones y podrán cesar, además de por las causas habituales, también por el transcurso del plazo para el que fueron nombrados ${ }^{4}$.

Por ello, resulta interesante analizar si estas características existentes entre los 2 principales grupos de jueces pueden provocar alguna diferencia en la forma de resolver los asuntos que tratan unos y otros. $Y$ esto es en lo que centraremos la parte empírica del artículo.

\section{Estado de la cuestión y antecedentes bibliográficos}

El análisis del comportamiento de los jueces en sus decisiones ha venido tratándose ya en la literatura académica, tanto desde el punto de vista psicológico como sociológico y puramente económico. Así pues, estudiar el comportamiento de los jueces y los factores que pueden influir en sus deliberaciones trasciende el plano analítico, dado que afecta a uno de los pilares fundamentales que vertebra un país: la Justicia. El funcionamiento de los órganos judiciales es, por tanto, una pieza fundamental de una sociedad. Pero también es importante señalar que, al igual que existen factores políticos o sociales que puedan afectar a las decisiones judiciales, el aspecto económico no es menos significativo, tal y como se ha tratado ya en algunas investigaciones previas aplicadas a otros países (Macis, 2001; Ichino et al., 2003; Mora, 2006, y Marinescu, 2011).

Existen trabajos llevados a cabo sobre el análisis del comportamiento de los jueces desde una perspectiva más general. Ya desde la publicación del clásico trabajo de Tversky y Kahneman (1974) sobre las reglas cognitivas que se aplican en las tomas de decisiones, han sido numerosos los trabajos que se han centrado en el comportamiento judicial. Muñoz Aranguren (2011) ha desarrollado recientemente esta idea para España.

Bornstein y Miller (2008) resumen las principales investigaciones sobre la relación entre la religión del juez y sus decisiones judiciales, en cuanto que aquella tiende a configurar la actitud personal y moral de dicho juez y, por ello, podría también motivar de distinto modo los fallos que

\footnotetext{
${ }^{4}$ Aunque las funciones de un juez sustituto son las mismas que las del juez titular, sus condiciones laborales no son iguales. Su forma de ingreso tiene 2 fases. La primera de ellas consiste en hacer un dictamen sobre el orden jurisdiccional elegido a partir de un caso práctico propuesto. Una vez superada esta prueba, la segunda fase consiste en una entrevista personal donde se consigue la habilitación para la función propuesta. No obstante, el juez suplente no ha superado una oposición de acceso a la Función Pública, y por tanto no posee todos los requisitos para ser un juez titular. En este sentido, en los entornos jurídicos se puede considerar que los jueces sustitutos presentan un menor grado de profesionalidad que los titulares.
} 
llevara a cabo. Yoon (2006) menciona la existencia de factores institucionales y personales, tales como la edad, el entorno político y el tipo de pensión, que influyen en las bajas de los jueces. $Y$ se plantea la posibilidad de que si todos estos factores afectan al servicio judicial, podrían también influir en las decisiones de esos jueces cuando están activos.

También desde la «economía del comportamiento» se han aportado recientemente algunas ideas. Baum (2010) se centró en los factores psicológicos que motivan a los jueces en cuanto que son agentes decisores. Viscusi (1999) profundiza en los factores que pueden influir a los jueces derivados de su actitud frente al riesgo y la incertidumbre del proceso de toma de decisiones. En la misma línea también podemos encontrar el trabajo de Rachlinski (1996).

En nuestro país, Manzanos (2004) ha estudiado los factores extrajudiciales que inciden en las decisiones de los jueces, y que divide en varios grupos: factores sociales y profesionales (derivados de su funcionarización); factores ideológicos, religiosos y culturales; factores subjetivos como la edad, el sexo, el estado civil o la educación recibida; factores relacionados con la presión mediática, o incluso el hecho de quién sea el propio procesado.

Las causas de las decisiones de los jueces han sido estudiadas también desde un ámbito económico. Profundizando en la relación entre Derecho y Economía, hay que mencionar algunas investigaciones que han abordado esta cuestión desde la perspectiva del AED. Cooter y Rubinfeld (1989) incorporaron un modelo de utilidad económica para estudiar las decisiones judiciales, tanto en el proceso previo como en la resolución. Burgess et al. (2001) analizan el incremento de casos judiciales en los tribunales de lo Social norteamericanos estudiando los factores que afectan a la decisión del trabajador, que sigue un análisis coste-beneficio, a la hora de reclamar. Entre estos factores incluyen la expectativa de ganar el juicio.

Pero también existe alguna evidencia previa de la influencia de las condiciones económicas en los jueces. Los recientes trabajos de Brennan et al. (2009a, b) arrojan algo de luz en esta dirección. En el primero, replicando el comportamiento de los jueces como si fueran votantes de unas elecciones políticas, comprueban que siguen un comportamiento cortoplacista y predicen, a través de un modelo econométrico con datos para la primera mitad del sigloxx, que las épocas recesivas y expansivas de la economía les influyen del mismo modo que lo hacen en los votantes con respecto al apoyo que prestan o no al Gobierno del país. En el segundo trabajo profundizan en el estudio de los condicionantes macroeconómicos de las decisiones judiciales de la Corte Suprema de Estados Unidos en los casos en que una de las partes es alguna de las instituciones estatales.

Por último, cabe también hacer referencia a los trabajos que centraron el análisis económico de las decisiones de los jueces en el ámbito concreto de los casos de despido ${ }^{5}$.

\footnotetext{
${ }^{5}$ Una literatura muy relacionada con esta sería la que desde el campo de la EL analiza los efectos de cambios en alguna medida de los costes de despido sobre las principales magnitudes laborales; por ejemplo, la tasa de ocupación, la tasa de desempleo o la tasa de actividad. Una revisión muy reciente de este tipo de trabajos puede encontrarse en Frick et al. (2012). Un interesante trabajo teórico
}

Ichino et al. (2003) han estudiado esta relación para los casos de despido de una empresa en Italia a partir de un trabajo previo de Macis (2001). Encuentran, sin embargo, conclusiones distintas. Ichino et al. (2003) evidencian que un mayor desempleo se asocia con mayor número de victorias de los trabajadores en sus pleitos, lo que concluyen tiene que ver con el hecho de que cuando las condiciones laborales son más adversas, los jueces tienden a ser más favorables a los trabajadores. Este resultado, además de ser significativo, también es importante, ya que demuestran que un incremento de un punto porcentual en la tasa de desempleo hace descender la probabilidad de que la firma gane el caso en 2,5 puntos porcentuales. Además, encuentran en consonancia sus resultados con la tesis de Donohue y Siegelman (1991), quienes sugieren que los trabajadores son más proclives a utilizar el sistema judicial en épocas recesivas si su situación está amenazada.

En cambio, Marinescu (2011) recientemente ha demostrado que en Gran Bretaña el aumento de la tasa de desempleo y la tasa de quiebra de empresas hace descender la probabilidad de que los jueces sentencien a favor del trabajador. Basándose en la investigación de Farber y Bazerman (1986), aducen que los árbitros son sensibles a las empresas en las épocas recesivas. Así pues, demuestran que las condiciones económicas influyen a los jueces tanto directamente (en cuanto que es un elemento que toman en consideración) como indirectamente (a través del comportamiento de los trabajadores y empresas antes del conflicto), lo que relacionan con la «calidad del caso». En el mismo sentido, un trabajo previo de Marinescu (2003) demostró que el desempleo influía negativamente en el porcentaje de victorias de los trabajadores con datos para Francia.

Para datos españoles, Bentolila (1997) presenta el coste esperado de despido como un resultado de la respuesta estratégica que tienen las empresas y los trabajadores ante el marco legislativo de referencia, especialmente en los casos en que no existe acuerdo entre las partes. Los jueces crearían así un marco de incentivos que operarían en el mercado de trabajo, afectando a dicho coste esperado a través de una vía directa (aumentando la proporción de casos en los que se declara improcedente o nulo, aumentando la demora en la resolución, y aumentando el coste de asesoramiento legal del empresario), y de una forma indirecta, a través de la probabilidad de que el trabajador recurra el despido, la probabilidad de conciliación previa y la disposición del empresario a pagar mayor indemnización pactada previamente al juicio, además de la incertidumbre que genera el proceso de reclamación. Por su parte, Mora (2006) estima paneles dinámicos con datos de comunidades autónomas para el periodo 1997-2002 y encuentra que existe una relación positiva entre la tasa de paro y el porcentaje de asuntos ganados por los trabajadores. En este mismo trabajo también hay efectos significativos de conflictividad laboral (huelgas realizadas) y de la predisposición de los jueces a fallar a favor de los trabajadores.

Así pues, es evidente que los pocos trabajos previos que existen en esta línea llegan a conclusiones en ocasiones

que analiza los efectos macroeconómicos de los costes de despido desde la perspectiva de los salarios de eficiencia es Galdón-Sánchez y Güell (2003). 
opuestas. Por ello, resulta interesante comprobar qué ocurre en España, en un periodo en el que se ha producido una crisis de unas proporciones no conocidas en la historia reciente, y especialmente constatar si existen diferencias entre los jueces titulares del juzgado y los sustitutos.

\section{Coste de despido y decisiones judiciales}

Muchos de los trabajos académicos sobre costes de despido dentro de la disciplina de la EL tienden a considerar que dicho coste viene prefijado por la legislación recogida en el derecho del trabajo. En este sentido, los trabajos de Gabuthy y Lambert (2008) y Goerke y Pannenberg (2010) recopilan la literatura internacional más destacada sobre la influencia de la regulación de los costes de despido ${ }^{6}$. De este modo, cuando se quieren determinar los efectos de dicha variable sobre otra magnitud como la tasa de desempleo o la tasa de ocupación, tienden a buscar cambios normativos de dicha legislación en el tiempo, dentro de una misma unidad territorial (generalmente un país o una región), comparar diferentes legislaciones en distintos territorios en un momento del tiempo o una mezcla de las 2 estrategias empíricas (utilizando, por ejemplo, la metodología de los datos de panel).

En este trabajo, no obstante, se pretende conectar la literatura académica anterior con un planteamiento más propio del campo del AED. Más precisamente, se profundizará en cómo las decisiones judiciales afectan a los costes de despido. Para entender esto mejor, en este apartado esbozaremos un marco teórico muy simple que pone de manifiesto este punto. Dado que esta es una investigación eminentemente empírica, la teoría presentada en este epígrafe se mantendrá en el nivel más sencillo posible para transmitir con claridad la idea central: un aumento en la probabilidad de fallo a favor del trabajador aumenta los costes efectivos de despido para el empleador. La clave está en que existe un espacio para la negociación entre trabajador y empresario, y el poder de negociación del trabajador aumenta si se sabe protegido por el sistema judicial. Dos trabajos esencialmente teóricos que profundizan mucho más en esta cuestión son los de Malo (2000) y Malo y Pérez (2003).

Sin entrar en tantos detalles como los trabajos arriba citados, el primer aspecto que hay que destacar en nuestro modelo es que el coste de despido no es siempre de una cuantía cierta y determinada, sino que podría depender de un fallo judicial. Para simplificar, consideremos que un juez de lo Social que resuelve un caso de despido puede fallar a favor o en contra del trabajador. Esos 2 estados

\footnotetext{
${ }^{6}$ En el trabajo de Gabuthy y Lambert (2008) se analiza la literatura sobre legislación de protección al empleo centrada en los despidos individuales, y el efecto que genera sobre el comportamiento de los agentes involucrados. Comprueban que la normativa legal no solo influye en el comportamiento de las partes en el momento de la ruptura contractual, sino también a lo largo de la relación laboral. Por otro lado, Goerke y Pannenberg (2010) elaboran un modelo teórico que predice que tanto el coste resultante de una reclamación de un trabajador como los factores legales que dicha reclamación conlleva afectan a la incidencia del coste de despido. Sin embargo, su nivel solo varía por medio de regulación legal. Para ello, emplean un panel de datos de 1991 a 2006 en Alemania oeste.
}

de la naturaleza condicionan el resultado final de la extinción de la relación contractual por parte del empresario. Supongamos que un fallo a favor del trabajador (en contra del empresario) supone un coste de despido de cuantía $\alpha$. Por otra parte, si el juez falla en contra del trabajador (a favor del empresario), el monto del despido es de cuantía $\beta$. Ante esta tesitura, el empleador podría estratégicamente ofrecer al trabajador una indemnización de cuantía $\delta$ con el objetivo de que este último aceptara la oferta y no llevara el caso a la vía judicial. Parece lógico, pues, considerar que $\alpha>\delta>\beta$. Como consecuencia de todo ello, el resultado final es que el coste de despido $(C)$ debe conceptualizarse como un coste esperado y que puede resumirse a través de la expresión (1):

$$
C=q(\delta, X) \delta+(1-q(\delta, X))(p(Z) \alpha+(1-p(Z)) \beta+\gamma)
$$

En la expresión (1), $q(\delta, X)$ hace referencia a la probabilidad de que el trabajador acepte la oferta de indemnización del empresario y no lleve el asunto a la vía judicial, y depende de un vector genérico de variables que podrían recoger características personales del trabajador $\mathrm{y} / \mathrm{o}$ del puesto de trabajo $(X)$ y de la magnitud de la indemnización propuesta por el empleador $(\delta)$. Con respecto a esta segunda cuestión, cuanto más alta sea la indemnización ofrecida por el empresario, más probable es que el trabajador la acepte ${ }^{7}$ (y más improbable es que se inicie una vía judicial). En términos formales: $q_{\delta}=\partial q(\cdot) / \partial \delta>0$.

Por otro lado, si el caso llega a juicio, con probabilidad $1-q(\cdot)$, el juez puede fallar a favor del trabajador con probabilidad $p(Z)$, otorgándole una cuantía $\alpha$, siendo $Z$ un vector de factores condicionantes de dicha decisión ${ }^{8}$. Evidentemente, con probabilidad $1-p(Z)$ el juez falla contra el trabajador y este recibe una cantidad $\beta$. Finalmente, $\gamma$ hace referencia a los costes relativos al proceso judicial (tasas, remuneración de abogados, salarios de tramitación, etc.).

Del marco teórico anterior se deduce que la variable de elección clave para el empresario (y que determina el coste efectivo del despido) es la indemnización $\delta$. En otras palabras, el empleador debe seleccionar una indemnización $\left(\delta^{*}\right)$ que minimice el coste esperado del despido recogido en la expresión (1). La condición de primer orden de mínimo se puede resumir por medio de (2):

$$
\frac{\partial C}{\partial \delta}=q_{\delta} \delta+q-q_{\delta}(p \alpha+(1-p) \beta+\gamma)=0
$$

La condición de segundo orden de mínimo exigiría que la derivada de la expresión (2) con respecto a $\delta$ tuviera signo positivo. Esto es lo que se recoge en (3):

$$
\frac{\partial^{2} C}{\partial \delta^{2}}=\Omega=q_{\delta \delta} \delta+2 q_{\delta}-q_{\delta \delta}(p \alpha+(1-p) \beta+\gamma)>0
$$

Una vez que se ha determinado la indemnización óptima $\left(\delta^{*}\right)$, es interesante calcular cómo cambia esta ante cambios

\footnotetext{
7 Implícitamente, al adoptar este supuesto se está considerando que el trabajador reacciona ante la oferta del empresario y que dicho empresario tiene en cuenta dicha reacción a la hora de hacer la oferta de indemnización. En otras palabras, se está considerando un equilibrio de Stackelberg.

8 Por ejemplo, en este trabajo estamos especialmente interesados en el hecho de si el juez es titular del juzgado o suplente.
} 
en la probabilidad de que un juez falle a favor del trabajador en un asunto de despido. Para ello ha de tenerse en cuenta que la expresión (2) define una función implícita que relaciona (entre otras variables) $\delta$ y $p$ :

$G\left(\delta^{*}, p\right)=q_{\delta} \delta+q-q_{\delta}(p \alpha+(1-p) \beta+\gamma)$

Aplicando el teorema de la función implícita en (4) podemos obtener el efecto de un cambio en la probabilidad de que el juez falle a favor del trabajador sobre la indemnización óptima que debe ofrecer el empleador:

$\frac{\partial \delta^{*}}{\partial p}=-\frac{\partial G / \partial p}{\partial G / \partial \delta^{*}}=\frac{q_{\delta}(\alpha-\beta)}{\Omega}>0$

El signo positivo de la expresión (5) es debido a 3 hechos. En primer lugar, por hipótesis $q_{\delta}>0$. En segundo lugar, $\alpha>\beta$. Finalmente, el denominador $(\Omega)$ es positivo debido al cumplimiento de la condición de segundo orden. Lógicamente, esto significa que si $p(\cdot)$ aumenta, los costes de despido efectivos para el empleador se ven incrementados.

La utilidad de la expresión (5) para los fines de este trabajo consiste en poner de manifiesto que más allá de los límites fijados en la normativa laboral, y que en nuestro modelo vendrían recogidos por los parámetros $\alpha$ y $\beta$, hay otros factores determinantes del coste de despido que el empleador tiene que afrontar de manera efectiva. Aquí hemos destacado el efecto de las decisiones judiciales, puesto que será la variable objeto de estudio en las secciones empíricas de este artículo.

\section{Base de datos}

Los datos empleados en este trabajo provienen de la información registrada a nivel de juzgado que proporciona la estadística del CGPJ $^{9}$. Hasta el tercer trimestre de 2009, estos datos procedían de la información contenida en los cuestionarios estadísticos que cumplimentaban trimestralmente los Juzgados de lo Social y que se remitían a la Subdirección General de Estadística de este Ministerio como unidad responsable de la elaboración de la estadística. Sin embargo, a partir del cuarto trimestre de 2009, y como resultado del Convenio de colaboración firmado el 21 de julio de 2008 entre el CGPJ y el Ministerio de Trabajo e Inmigración, la citada información es recogida a través de cuestionarios web por el CGPJ, el cual, con posterioridad a una primera depuración, la remite en fichero a la Subdirección General de Estadística. En esta base de datos se recoge el flujo de asuntos que se produce en todos los Juzgados de lo Social españoles desde el año 1995. No obstante, esta fuente solo aparece con toda la información requerida para este artículo desde el año 2004, con la inclusión de desagregaciones referidas al sentido de la sentencia (estimación o desestimación), al tipo de magistrado (titular, sustituto, refuerzo o titular de otro juzgado) o a la materia del conflicto (despidos, reclamaciones por cantidades, Seguridad Social y conflictos colectivos).

Dado que el objetivo fundamental del estudio es la comparación del grado de estimación de asuntos entre jueces

\footnotetext{
${ }^{9}$ Disponible en: http://www.poderjudicial.es/cgpj/es/Temas/ Estadistica_Judicial
}

titulares y suplentes ${ }^{10}$, el periodo muestral es el comprendido entre 2004 (que es el primer año en el que hay desagregaciones por sentido de la sentencia y tipo de juez) hasta 2011, que es el último del que se dispone de información. Este periodo es también idóneo para estudiar la importancia del ciclo económico, ya que incluye los 4 últimos años de la fase expansiva (2004-2007) y los 4 primeros de la recesiva (2008-2011). Finalmente, de los 4 tipos de asuntos resueltos que se recogen en la estadística se seleccionan solo los individuales referidos a temas de despido, debido al objeto del trabajo. Además, en este tipo de asuntos queda claro quién es el beneficiario de la sentencia. La estimación de un asunto de despido siempre es a favor del trabajador, mientras que la desestimación es a favor del empresario. Por lo tanto, una mayor estimación de asuntos puede relacionarse con una mayor sensibilidad hacia la situación del trabajador y, por consiguiente, un aumento de los costes de despido.

Un aspecto importante de cualquier investigación es conocer cuál es la magnitud del fenómeno analizado. Con esta finalidad se construye la tabla 1, donde se presenta la información referida a la importancia que tienen los asuntos de despido resueltos dentro del total de asuntos que llegan al tribunal de lo Social y el peso que tienen las decisiones de los jueces suplentes en relación con los titulares en asuntos de despido. Así, por ejemplo, en 2011 los juzgados de lo social españoles resolvieron más de 175.000 asuntos, de los cuales cerca de la cuarta parte se referían a temas individuales de despido resueltos por jueces titulares o suplentes. Concretamente, los jueces titulares emitieron 36.006 sentencias, mientras que los suplentes resolvieron 8.659 asuntos (cerca del $20 \%$ del total resuelto por estos tipos de juez).

Un problema que se plantea es que en algunos de los juzgados no hay asuntos resueltos por ambos tipos de juez. Para solucionar en cierta medida esta deficiencia se recurre a realizar una agregación de asuntos a nivel provincial, y de esta manera se consigue una muestra de 50 provincias (se eliminan las ciudades autónomas Ceuta y Melilla) para 8 años (4 de crisis económica y 4 previos a ella). No obstante, sigue habiendo provincias en las que para algunos años no hay sentencias emitidas por jueces titulares o sustitutos, y por lo tanto los paneles resultantes serán no balanceados. Además, y con el fin de evitar los problemas que pueden surgir cuando hay muy pocas sentencias, se han eliminado todos los datos de provincias donde el número de resoluciones es inferior a $20^{11}$.

\footnotetext{
10 Se ha decidido utilizar solo estos 2 tipos de juez porque el número de asuntos que resuelven el resto es muy pequeño. La otra alternativa era unirlos a los jueces suplentes como contraposición a los titulares, pero estaríamos introduciendo ruido en el modelo y nos alejaríamos del objetivo inicial de esta investigación.

$11 \mathrm{Si}$ en un año y provincia concretos los asuntos resueltos por un tipo de juez son menos de 20, ese dato se elimina de la muestra. El objetivo de esta corrección es evitar los casos extremos cuando se calculan porcentajes de estimación con pocas sentencias. Se ha decidido utilizar el límite de 20 asuntos porque así el número de datos para los 2 tipos de juez supera los 250 y porque los test aplicados a posteriori reconocen la diferencia de momentos y de distribución entre los 2 tipos de juez.
} 
Tabla 1 Número de asuntos de despido resueltos según tipo de juez por año y su relación con los asuntos sociales

\begin{tabular}{|c|c|c|c|c|c|}
\hline & \multirow[t]{2}{*}{ Asuntos resueltos en Tribunal de lo Social (3) } & \multicolumn{3}{|c|}{ Asuntos de despidos resueltos } & \multirow[t]{2}{*}[(1)+(2)]{$/(3)$} \\
\hline & & Titulares (1) & Sustitutos (2) & $(2) /[(1)+(2)]$ & \\
\hline 2004 & 160.507 & 25.496 & 4.211 & $14,2 \%$ & $19 \%$ \\
\hline 2005 & 157.469 & 26.500 & 3.654 & $12,1 \%$ & $19 \%$ \\
\hline 2006 & 155.245 & 26.832 & 3.720 & $12,2 \%$ & $20 \%$ \\
\hline 2007 & 153.367 & 26.550 & 3.371 & $11,3 \%$ & $20 \%$ \\
\hline 2008 & 160.039 & 37.465 & 4.686 & $11,1 \%$ & $26 \%$ \\
\hline 2009 & 184.677 & 50.900 & 9.857 & $16,2 \%$ & $33 \%$ \\
\hline 2010 & 178.662 & 33.619 & 6.925 & $17,1 \%$ & $23 \%$ \\
\hline 2011 & 176.844 & 36.006 & 8.659 & $19,4 \%$ & $25 \%$ \\
\hline
\end{tabular}

Fuente: Elaboración propia a partir de la información procedente de las estadísticas del CGPJ.

Tabla 2 Porcentaje de asuntos estimados por tipo de juez, provincia y año (más de 20 asuntos resueltos)

\begin{tabular}{|c|c|c|c|c|c|c|}
\hline \multirow[b]{2}{*}{ Año } & \multicolumn{3}{|c|}{ Jueces titulares } & \multicolumn{3}{|c|}{ Jueces sustitutos } \\
\hline & Media & Desviación & Provincias & Media & Desviación & Provincias \\
\hline 2004 & 0,706 & 0,077 & 49 & 0,738 & 0,120 & 32 \\
\hline 2005 & 0,697 & 0,074 & 49 & 0,703 & 0,081 & 24 \\
\hline 2006 & 0,702 & 0,064 & 50 & 0,698 & 0,089 & 27 \\
\hline 2007 & 0,710 & 0,070 & 48 & 0,729 & 0,069 & 29 \\
\hline 2008 & 0,751 & 0,064 & 48 & 0,739 & 0,114 & 36 \\
\hline 2009 & 0,770 & 0,069 & 50 & 0,749 & 0,098 & 37 \\
\hline 2010 & 0,744 & 0,062 & 50 & 0,723 & 0,082 & 36 \\
\hline 2011 & 0,732 & 0,066 & 50 & 0,708 & 0,088 & 40 \\
\hline Total & 0,727 & 0,072 & 394 & 0,725 & 0,095 & 261 \\
\hline
\end{tabular}

Nota: En el apéndice 1 se muestra una relación de los datos eliminados por año y tipo de juez.

Fuente: Elaboración propia a partir de la Estadística del CGPJ.

\section{Análisis descriptivo de las diferencias según el tipo de juez}

Una primera pregunta a responder es si tiene sentido considerar que existe un comportamiento diferenciado entre jueces titulares y suplentes a la hora de estimar asuntos de despido. Para dar respuesta a esta cuestión se plantea un triple análisis, eminentemente descriptivo, que pone de manifiesto algunas regularidades empíricas de gran interés. En primer lugar se analizan los estadísticos descriptivos de las 2 poblaciones; en segundo lugar se implementan algunos test de igualdad de los distintos momentos de la muestra y del total de la distribución, y en tercer lugar se estudia el gráfico Kernel de las 2 distribuciones.

En la tabla 2 se muestran la media y la desviación típica del porcentaje de asuntos de despido estimados por tipo de juez, provincia y año cuando se resuelvan más de 20 asuntos. Un primer resultado a tener en cuenta es que la media del porcentaje de asuntos de despido estimados por provincia es muy similar en los 2 tipos de juez cuando se usa la muestra agregada, pero presenta diferencias si realizamos el análisis año a año, con brechas que superan, por ejemplo, los 3 puntos porcentuales en 2004. Este mismo resultado se puede deducir también de las evoluciones que presenta el porcentaje de estimación de asuntos que se muestra en la figura 2 Concretamente, se aprecia una mayor estimación de los suplentes en el periodo previo a la crisis (salvo en

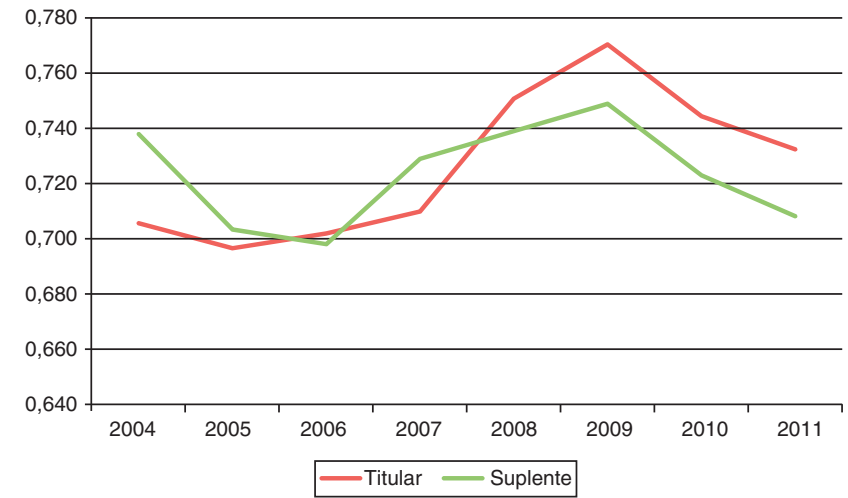

Figura 2 Evolución del porcentaje de asuntos estimados por tipo de juez.

Fuente: Elaboración propia a partir de la Estadística del CGPJ.

el año 2006, donde los porcentajes son muy similares) y de los titulares durante los años de recesión. A la vista de la tabla 2, también se comprueba que durante todo el periodo muestral, tanto en el dato total como en los de cada uno de los años, las desviaciones con respecto a los valores medios son sensiblemente superiores en el caso de los jueces suplentes que en el de los titulares. Este resultado parece indicar que existe una mayor aleatoriedad en el comportamiento de los primeros y que su valor medio es menos representativo. 


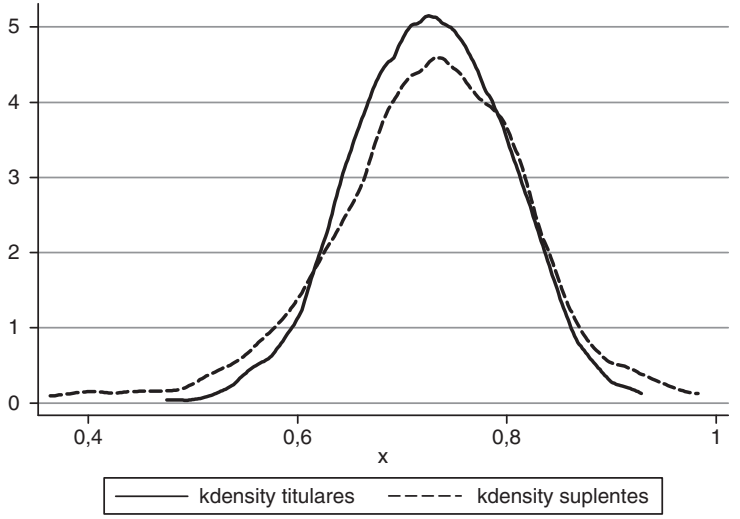

Figura 3 Distribución del porcentaje de asuntos estimados en cada provincia y año por tipo de juez (más de 20 asuntos resueltos).

Fuente: Elaboración propia a partir de la Estadística del CGPJ.

Para corroborar estas primeras intuiciones se aplican los correspondientes test de igualdad de medias, de igualdad de varianzas y de igualdad de distribuciones. La igualdad de momentos se rechaza con un nivel de significación del $10 \%$ en el caso de las medias y del $1 \%$ en el caso de las varianzas. Para la igualdad de distribuciones se utiliza el test no paramétrico Epps-Singleton que, de acuerdo con Goerg y Kaiser (2009), es usualmente más potente que el test de Kolmogorov-Smirnov para demostrar si los datos provienen de poblaciones diferentes. Este test también rechaza la igualdad de distribuciones con un nivel de confianza del $5 \%{ }^{12}$.

Finalmente, la figura 3 presenta el gráfico Kernel del porcentaje de asuntos estimados por tipo de juez y refleja de una forma visual la diferencia entre las 2 distribuciones. Nuevamente se comprueba cierta similitud en las medias pero con una dispersión superior en el caso de los jueces suplentes que refleja una mayor aleatoriedad en el comportamiento de este colectivo. La misma conclusión se extrae si el Kernel se realiza por separado en las 2 fases del ciclo y también si se estudian los años de forma independiente. Este resultado, junto con todo lo concluido en los párrafos previos, parece dejar claro que existen diferencias significativas en el porcentaje de asuntos de despido resueltos a favor del trabajador en función de si el juez es titular o suplente. En el siguiente apartado se profundiza en esta cuestión utilizando técnicas de análisis econométrico multivariante.

\section{Análisis de las diferencias con técnicas de regresión}

Desde un punto de vista metodológico, la técnica utilizada para estudiar estas diferencias en el comportamiento en función del tipo de juez es la estimación de 2 paneles

\footnotetext{
12 Los resultados de todos estos test están a disposición de los interesados previa petición a los autores. Estos mismos test también se han aplicado para comprobar la desigualdad de medias, varianzas y distribuciones a nivel de juzgado cuando los asuntos resueltos son superiores a 20 , y en todos ellos también se rechaza la igualdad entre jueces titulares y suplentes.
}

provinciales no balanceados con efectos fijos. Se considera que es más adecuado emplear efectos fijos porque las observaciones utilizadas se corresponden con el total de la población y no son una muestra de una población mayor. No obstante, esta elección se justifica de forma detallada más adelante.

La estimación base del modelo viene dada por la siguiente expresión:

$p_{i, t}^{j}=k^{j}+Z_{i, t}^{j} \theta^{j}+\mu_{i, t}^{j}$

donde $\kappa$ es una constante, $Z_{i, t}^{\prime}$ es un vector de variables explicativas con variabilidad espacial y temporal, y $\theta$ es el conjunto de parámetros a estimar. Nuestra variable dependiente, $p_{i, t}$, se refiere al porcentaje de asuntos resueltos por el juez que acaban con un fallo a favor del trabajador. Por su parte, el superíndice $j$ indica el grupo poblacional analizado (jueces titulares o suplentes), el subíndice $i$ refleja la dimensión cross-section (provincias españolas) y el subíndice $t$ la dimensión temporal (años). Además, y dado que disponemos de datos de panel, el término de error se descompone de la siguiente manera.

$\mu_{i, t}^{j}=u_{i}^{j}+v_{i, t}^{j}$

siendo $u_{i}$ el efecto específico individual y $v_{i, t}$ la perturbación aleatoria restante.

Como ya se ha comentado en un párrafo anterior, el punto de partida del análisis econométrico es la estimación de 2 paneles no balanceados, uno para los jueces titulares y otro para los sustitutos, donde la variable dependiente es el porcentaje de asuntos de despido que se resuelven a favor del trabajador. Esa variable dependiente se tratará de explicar a través de varios regresores.

El primero de dichos regresores es la tasa de paro, que se incluye como indicador del ciclo económico. Como se ha comentado en una sección previa, hay trabajos que encuentran una relación directa entre el desempleo y los fallos a favor del trabajador (Ichino et al., 2003, y Mora, 2006) y otros que encuentran la relación contraria (Marinescu, 2003, y Marinescu, 2011). Debido a esta disparidad en los resultados, pensamos que podría existir algún tipo de asimetría cíclica, y por ello en algunas especificaciones econométricas también se introduce la tasa de paro multiplicada por una dummy que toma el valor 1 en los años de crisis (esto es, de 2008 a 2011).

Incluimos también una variable que intenta recoger el impacto de la reforma legislativa aprobada por la ley $35 / 2010$ de 17 de septiembre, en la que se ampliaban las causas por las que se podía reconocer un despido como procedente. Esta variable toma el valor 0,3 en 2010 (el 0,3 indica que la ley está en vigor el $30 \%$ de los días de ese año), 1 durante 2011 y cero en el resto de la muestra ${ }^{13}$. Investigaciones previas han puesto de manifiesto ${ }^{14}$ que el marco normativo del derecho del trabajo afecta a variables

\footnotetext{
13 También se han realizado estimaciones donde solo se consideran efectos de la ley en 2011. De esta manera se da más robustez a los resultados y se consigue tener en cuenta la dilación en la llegada de los asuntos al juzgado.

14 Así, el trabajo de García-Martínez y Malo (2007) examina los efectos de algunas reformas laborales sobre los despidos individuales y colectivos, mientras que el trabajo de Frick et al. (2012)
} 
Tabla 3 Resultados de la estimación del panel de efectos fijos

\begin{tabular}{|c|c|c|c|c|c|c|}
\hline Modelo & 1 & $\|$ & III & IV & v & $\mathrm{VI}$ \\
\hline \multicolumn{7}{|l|}{ Titular } \\
\hline Tasa de paro & $0,278^{* * *}$ & $-0,285^{* * *}$ & $0,389^{* * *}$ & $-0,183^{*}$ & $0,408^{* * *}$ & $-0,162^{*}$ \\
\hline Tasa de paro $\times$ crisis & & $0,372^{* * *}$ & & $0,402^{* * *}$ & & $0,398^{* * * *}$ \\
\hline Ley $35 / 2010$ & & & $-0,028^{* * *}$ & $-0,037^{* * *}$ & $-0,037^{* * *}$ & $-0,045^{* * *}$ \\
\hline Carga del juzgado & & & & & $-0,003^{*}$ & $-0,003^{*}$ \\
\hline Constante & $0,690^{* * * *}$ & $0,732^{* * * *}$ & $0,680^{* * * *}$ & $0,722^{* * *}$ & $0,698^{* *+* *}$ & $730^{* * *}$ \\
\hline \multicolumn{7}{|l|}{ Sustituto } \\
\hline Tasa de paro & 0,066 & 0,004 & $0,257^{* *}$ & 0,164 & $0,276^{* *}$ & 0,198 \\
\hline Tasa de paro $\times$ crisis & & 0,043 & & 0,065 & & 0,054 \\
\hline Ley $35 / 2010$ & & & $-0,046^{* *}$ & $-0,047^{* *}$ & $-0,055^{* *}$ & $-0,055^{* * *}$ \\
\hline Carga del juzgado & & & & & $-0,003$ & $-0,002$ \\
\hline Constante & $0,716^{* * *}$ & $0,720^{* * *}$ & $0,698^{* * *}$ & $0,705^{* * *}$ & $0,706^{* * *}$ & $0,711^{* * * *}$ \\
\hline
\end{tabular}

En todos los casos los test de la $\mathrm{F}$ demuestran la significación de los efectos fijos provinciales de forma conjunta.

Tanto la variable dependiente como la tasa de paro están expresadas en tantos por 1.

Fuente: Elaboración propia a partir de datos de las estadísticas del CGPJ y de la EPA.

" Significativo al $10 \%$.

** Significativo al $5 \%$.

*** Significativo al $1 \%$.

económicas involucradas en el proceso de extinción de los contratos laborales ${ }^{15}$. Dado el contenido de la reforma que nos concierne (y dada la definición de nuestra variable dependiente), esperamos un signo negativo asociado a dicha variable

Finalmente, también se incluye una variable que trata de medir el efecto saturación de trabajo en los juzgados. Este regresor se construye como cociente entre los asuntos pendientes al inicio del año y los resueltos por jueces titulares y suplentes durante ese ejercicio. Con esta variable pretendemos detectar si la carga de trabajo afecta a la forma de resolver los asuntos por parte del juez. No conocemos ninguna teoría que anticipe el signo de dicha variable, por lo que esta es una cuestión esencialmente empírica que vendrá determinada por los resultados de nuestra base de datos.

En la tabla 3 se presenta el valor y la significación estadística de los coeficientes estimados con un desglose según el tipo de juez y para 6 especificaciones econométricas diferentes, con el fin de comprobar la robustez de los resultados. Como ya se ha comentado previamente, las estimaciones de los 2 paneles no balanceados se realizan suponiendo efectos fijos porque, de acuerdo con la literatura, se suele considerar que cuando se tienen datos de todos las unidades individuales (en nuestro caso tenemos datos de todas las provincias), cada uno de ellos tiene su propio intercepto que debe ser estimado a través de efectos fijos ${ }^{16}$. En cuanto

analiza los efectos sobre la demanda de reclamaciones laborales individuales en materia de despido.

${ }^{15} \mathrm{Si}$ bien los factores más determinantes son los condicionantes económicos, como la tasa de desempleo.

16 No obstante, se han realizado estimaciones de efectos aleatorios. En todas ellas el test de Breuch y Pagan muestra que los efectos aleatorios son mejores que la regresión OLS. También se han aplicado los test de Hausman, que indican que no hay diferencias sistemáticas entre los coeficientes para el modelo v y no es concluyente para el modelovi; por lo tanto, este método también podría a las especificaciones econométricas, estas se corresponden con 6 modelos diferentes: el I y el II incluyen solo el efecto del ciclo económico, el III y el iv incluyen el ciclo y la ley aprobada en septiembre de 2010 , mientras que los modelos $\vee$ y vı añaden a las variables anteriores el efecto de la carga de asuntos del juzgado. Además, hay que apuntar que los modelos I, III y v incluyen solamente la tasa de paro como indicador del ciclo, mientras que los modelos II, IV y vi añaden la interacción de la tasa de desempleo con la dummy crisis con el fin de medir el efecto diferencial de la tasa de paro en los años de mala coyuntura económica ${ }^{17}$.

Los resultados de la tabla 3 permiten corroborar el diferente comportamiento de los jueces a la hora de estimar los asuntos de despido. En primer lugar se comprueba que los jueces titulares se muestran más sensibles a la evolución de la tasa de paro reconociendo un mayor porcentaje de despidos improcedentes, sobre todo en el periodo asociado a la crisis económica. Sin embargo, los magistrados suplentes, aunque también responden a la evolución del desempleo, lo hacen en menor medida y no muestran comportamiento diferencial asociado a la recesión. Este resultado es claro en el modelo vi, donde las variables que miden el desempleo no son significativas para la explicación de la tasa de estimación de los magistrados suplentes. Pero también se demuestra en el modelov, donde un incremento de 10 puntos en la tasa de paro genera un aumento de 4 puntos porcentuales en la tasa de estimación de asuntos de los titulares y de menos de 3 en el caso de los sustitutos. Por su parte, el hecho de

ser adecuado. Sin embargo, ni los coeficientes ni las significaciones sufren alteraciones sensibles. Los resultados de la estimación del panel con efectos aleatorios están a disposición de los interesados previa solicitud a los autores.

17 Se han realizado pruebas introduciendo una tendencia en el modelo completo. A nivel general, la tendencia elimina la significación de la variable que mide el desempleo pero no muestra ningún cambio reseñable en el resto de las variables. 
que los jueces suplentes no presenten un coeficiente significativo para la tasa de paro en el modelo I es consecuencia de que los efectos de esa variable se ven atenuados por el descenso final en la estimación debido al efecto de la ley $35 / 2010$, y por lo tanto es necesario que esta variable esté presente en el modelo.

En segundo lugar se puede apreciar que tanto jueces titulares como suplentes se ven influenciados por los efectos de la ley $35 / 2010$, reduciendo el porcentaje de despidos que consideran improcedentes ${ }^{18}$. No obstante, también se aprecia que esta reducción es sensiblemente mayor en el caso de los jueces suplentes. Así, el efecto de la ley produce una reducción de $4,5(3,7)$ puntos porcentuales en la tasa de estimación de los jueces titulares y de 5,5 puntos en la de los sustitutos para el último año de la muestra, cuando se estima el modelo $\mathrm{vI}(\mathrm{v})$. Este resultado es lógico porque se trata de una reforma legislativa que debe ser asumida por todos los jueces con independencia de cuál sea su situación administrativo-laboral.

Finalmente, y por lo que se refiere al efecto de la carga de trabajo en los juzgados, el hecho de que esta variable solo afecte a los jueces titulares y que no sea significativa para los magistrados suplentes parece poner de manifiesto una mayor implicación de este tipo de jueces con la situación de su juzgado. No obstante, el coeficiente de esta variable indica que es necesario un gran aumento del número de asuntos pendientes para que los efectos sobre la tasa de estimación sean apreciables.

Para obtener una idea del orden de magnitud de los efectos analizados se puede hacer un simple ejercicio de simulación. Si nos situamos en el periodo expansivo de la muestra en el que no está presente la ley, con una carga de juzgado media y suponiendo que la tasa de paro es la menor de toda la muestra (3,4\% en Tarragona en 2007), los jueces titulares estimarían un $69 \%$ (72\%) de las reclamaciones de los trabajadores de acuerdo a la especificación $v(\mathrm{vI})$. Sin embargo, los jueces suplentes estimarían el $71 \%$ de los casos en ambas especificaciones.

Si ahora repetimos el ejercicio en una situación de crisis económica y con la mayor tasa de paro posible (34\% en Almería en 2011$)$, los jueces titulares estimarían el $82 \%$ (80\%) de los casos con la especificación $v(\mathrm{vl})$ si no se ven afectados por la ley y el 78\% (76\%) si esta está presente. Sin embargo, los jueces suplentes pasarían a estimar el $78 \%$ de los casos con ambos modelos en ausencia de ley, y el 73\% cuando la ley $35 / 2010$ ya se ha aprobado

Para profundizar en esta cuestión, también se construye la simulación de la evolución de la tasa de estimación de asuntos de despido para jueces titulares y sustitutos cuando se emplea la tasa de paro en España con el modelo $v^{19}$. Los resultados que se observan en la figura 4 ponen de manifiesto una mayor estimación de asuntos en el

\footnotetext{
${ }^{18}$ Cuando solo se consideran los efectos de la ley durante 2011, los resultados son iguales en signo y significación. Se sigue cumpliendo que la ley afecta en mayor medida a los jueces suplentes, aunque su influencia global es algo menor. Para el caso del modelo completo el coeficiente pasa de 0,045 a 0,04 en el caso de los titulares y de 0,055 a 0,047 en el caso de los jueces sustitutos.

${ }^{19}$ Se utiliza el modelov porque en él la tasa de paro es significativa para los 2 tipos de juez. No obstante, la simulación con el
}

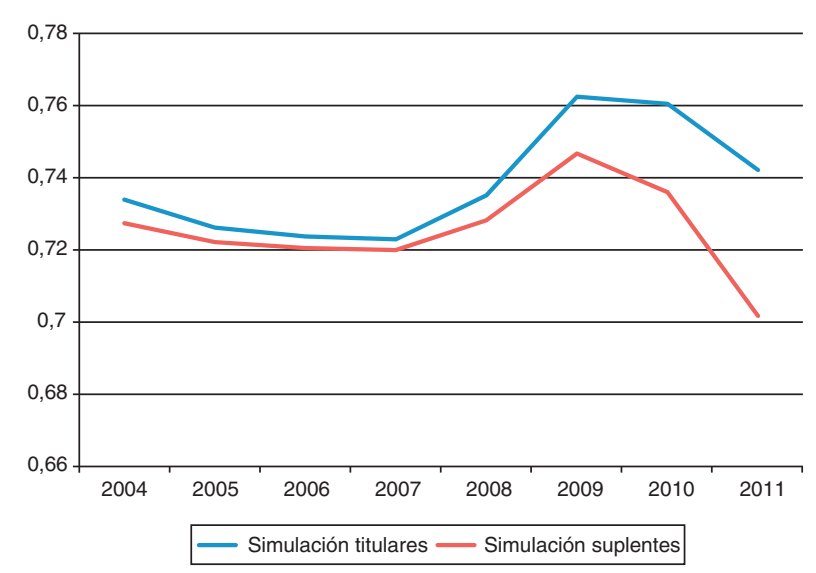

Figura 4 Simulación de la evolución del porcentaje de asuntos estimados por titulares y suplentes utilizando la tasa de paro de España (modelov).

Fuente: Elaboración propia a partir de la estimación del modelov.

caso de los jueces titulares que se agudiza durante el periodo de crisis y más en el periodo de vigencia de la ley 35/2010, donde la brecha llega a estar cercana a los 5 puntos porcentuales. Este resultado sin duda afecta también a los costes de despido, en el sentido de que los jueces titulares aumentan más los costes para el empleador en las fases recesivas por el hecho de fallar más a favor del trabajador.

La interpretación tentativa de estos resultados se puede obtener desde una doble perspectiva. La primera de estas vías proviene de la cualificación de los magistrados. El hecho de que los jueces titulares hayan superado una oposición libre o un concurso entre juristas de reconocida competencia, y que además hayan recibido un curso en la Escuela Judicial, les proporciona sin duda un mayor nivel de cualificación que puede explicar las diferencias en el grado de estimación y en las desviaciones. En este sentido, siempre es más deseable ser juzgado por un juez profesional, y las organizaciones judiciales deberían incidir en esta cualificación con el fin de reducir la incertidumbre en el resultado.

La segunda de las vías de explicación estaría relacionada con el llamado «sesgo cognitivo». Muñoz Aranguren (2011) identifica el origen de estos sesgos en procesos mentales de simplificación (heurística) a la hora de procesar la información procedente del exterior y presenta varias fuentes de sesgo, alguna de las cuales podría estar explicando las diferencias entre tipos de juez. Así, «el proceso heurístico de la representatividad» indica que el hecho de que los jueces suplentes resuelvan menos asuntos puede explicar la mayor dispersión de su grado de estimación de asuntos entre los diferentes juzgados. En esta misma línea, «el procedimiento de anclaje y ajuste» o «el sesgo retrospectivo» pueden explicar el hecho de que los jueces titulares respondan más al ciclo, en cuanto que tienen más información de la situación del entorno y unos precedentes que pueden influir en sus decisiones.

modelovi se ha realizado y está a disposición de los interesados previa solicitud a los autores. 


\section{Conclusiones}

Las decisiones de los jueces de lo Social son un componente importante de los costes de despido efectivo que afronta un empleador, y no es una dimensión que se haya tratado de forma extensa en la literatura. Este trabajo profundiza en esta cuestión y trata de dar respuesta a 2 cuestiones. La primera de ellas es si los jueces titulares y sustitutos tienen un comportamiento estadísticamente similar o diferente a la hora de estimar asuntos de despido. La segunda es si ambos tipos de jueces han modificado su comportamiento de forma similar o diferente al producirse el cambio cíclico durante la crisis más profunda de la historia reciente de España.

Mediante un análisis descriptivo se responde afirmativamente a la primera de esas cuestiones y se pone de manifiesto una mayor dispersión con respecto a la media en el porcentaje de asuntos que examinan los jueces sustitutos. Así, y de forma más detallada, se comprueba que el recorrido entre los percentiles 25 y 75 del porcentaje de asuntos de despido estimados es un $10 \%$ superior en jueces sustitutos que en titulares, y si lo ampliamos a los percentiles 10 y 90 , el recorrido es de 18 puntos porcentuales para los jueces titulares y de 22 para los sustitutos (un $22 \%$ superior).

En relación con la segunda de las cuestiones, también se obtiene una respuesta afirmativa. La simple representación gráfica del porcentaje de asuntos de despido estimados por juez y año ya pone de manifiesto que mientras en la fase expansiva del ciclo el grado de estimación de asuntos era muy similar para ambos tipos de juez (e incluso superior para los jueces suplentes), la situación cambia cuando se incrementa el nivel de desempleo como consecuencia de la crisis, y el porcentaje de estimación pasa a ser superior en el caso de los jueces titulares.

Este efecto del ciclo se corrobora a través del uso de técnicas de regresión estadística. Se comprueba que la tasa de paro tiene una mayor influencia en los jueces titulares, sobre todo en los años que se corresponden con el periodo de crisis económica. En estos años, un incremento de la tasa de paro aumenta más el porcentaje de asuntos resueltos a favor de los trabajadores en el caso de los jueces titulares que en el de los sustitutos. Además, también se puede observar que los supuestos aprobados por la ley 35/2010, que reducen el porcentaje de casos fallados a favor de los trabajadores, tienen una mayor influencia en las decisiones de los jueces suplentes. Los 2 hechos de forma conjunta generan una significativa brecha a favor de los jueces titulares en los últimos años de la muestra.

En cuanto al porqué de estos comportamientos diferenciales, el trabajo concluye con 2 respuestas tentativas que pueden afectar al grado de estimación de asuntos. En primer lugar, se identifica una diferencia de cualificación asociada a un menor nivel de exigencia para los magistrados suplentes que para los titulares. $Y$ en segundo lugar, se presenta la posible presencia de sesgos cognitivos que afectan de forma diferente a cada tipo de juez.

\section{Agradecimientos}

Los autores agradecen los comentarios realizados por el coordinador de este número, Miguel Ángel Malo, y por un evaluador anónimo, que han mejorado la calidad del artículo. También queremos mostrar nuestro agradecimiento a los Secretarios Judiciales de los Juzgados de lo Social números 1 y 2 de Valladolid, que han permitido resolver algunas cuestiones relativas a ciertos temas jurídicos. No obstante, la responsabilidad de los posibles errores que pudieran existir es exclusivamente de los autores.

\section{Apéndice 1. Relación de provincias eliminadas de los paneles no balanceados por año y tipo de juez}

\begin{tabular}{|c|c|c|}
\hline & Titulares & Sustitutos \\
\hline 2004 & Teruel & $\begin{array}{l}\text { Almería, Córdoba, Huelva, Huesca, } \\
\text { Teruel, Ávila, Burgos, Palencia, } \\
\text { Salamanca, Segovia, Soria, Valladolid, } \\
\text { Zamora, Guadalajara, Girona, Cáceres, } \\
\text { Murcia y La Rioja }\end{array}$ \\
\hline 2005 & Teruel & $\begin{array}{l}\text { Almería, Cádiz, Córdoba, Jaén, Málaga, } \\
\text { Huesca, Teruel, Cantabria, Ávila, } \\
\text { Burgos, León, Palencia, Salamanca, } \\
\text { Segovia, Soria, Valladolid, Zamora, } \\
\text { Ciudad Real, Cuenca, Guadalajara, } \\
\text { Tarragona, Badajoz, Cáceres, Lugo, } \\
\text { Murcia y La Rioja }\end{array}$ \\
\hline 2006 & & $\begin{array}{l}\text { Almería, Cádiz, Córdoba, Granada, } \\
\text { Huesca, Teruel, Baleares, Ávila, } \\
\text { Palencia, Salamanca, Segovia, Soria, } \\
\text { Valladolid, Zamora, Ciudad Real, } \\
\text { Guadalajara, Lleida, Badajoz, Cáceres, } \\
\text { Lugo, Ourense y La Rioja }\end{array}$ \\
\hline 2007 & $\begin{array}{l}\text { Teruel, } \\
\text { Cuenca }\end{array}$ & $\begin{array}{l}\text { Almería, Córdoba, Jaén, Huesca, Teruel, } \\
\text { Baleares, Ávila, Palencia, Salamanca, } \\
\text { Segovia, Soria, Valladolid, Zamora, } \\
\text { Ciudad Real, Guadalajara, Lleida, } \\
\text { Tarragona, Badajoz, Cáceres, Lugo } \\
\text { y Ourense }\end{array}$ \\
\hline 2008 & $\begin{array}{l}\text { Teruel, } \\
\text { Cuenca }\end{array}$ & $\begin{array}{l}\text { Córdoba, Granada, Huelva, Jaén, } \\
\text { Huesca, Teruel, Baleares, Burgos, } \\
\text { Segovia, Soria, Valladolid, Zamora, } \\
\text { Lleida y Cáceres }\end{array}$ \\
\hline 2009 & & $\begin{array}{l}\text { Huelva, Jaén, Huesca, Teruel, Cantabria, } \\
\text { Burgos, Palencia, Segovia, Soria, } \\
\text { Cuenca, Badajoz, Cáceres y Ourense }\end{array}$ \\
\hline 2010 & & $\begin{array}{l}\text { Cádiz, Huelva, Huesca, Teruel, Baleares, } \\
\text { Ávila, Burgos, Palencia, Segovia, Soria, } \\
\text { Zamora, Albacete, Cuenca y Ourense }\end{array}$ \\
\hline 2011 & & $\begin{array}{l}\text { Huelva, Huesca, Ávila, Burgos, Palencia, } \\
\text { Salamanca, Soria, Zamora, Cuenca } \\
\text { y Ourense }\end{array}$ \\
\hline
\end{tabular}

\section{Bibliografía}

Baum, L., 2010. Motivation and judicial behavior: Expanding the scope of inquiry. En: Klein, D., Mitchell, G. (Eds.), The Psychology of Judicial Decision Making. American Psichology-Law Society Series, pp. 3-26.

Bentolila, S., 1997. Las decisiones judiciales sobre despidos en España: una perspectiva económica. Cuadernos de Información Económica, 170-179, 128-129. 
Bornstein, B.H., Miller, M.K., 2008. Does a judge's religion influence decision making? Court Review 45, 112-115.

Brennan, T., Epstein, L., Staudt, N., 2009a. The political economy of judging. Minnesota Law Review 93, 1503-1524.

Brennan, T., Epstein, L., Staudt, N., 2009b. Economic trends and judicial outcomes: A macrotheory of the court. Duke Law Journal 58, 1191-1230.

Burgess, S., Propper, C., Wilson, D., 2001. Explaining The Growth in the Number of Applications to Industrial Tribunals, 1972-1997 Employment Relations Research Series. Departamento de Industria y Comercio, Londres.

Cooter, R.D., Rubinfeld, D.L., 1989. Economic analysis of legal disputes and their resolution. Journal of Economic Literature 27, 1067-1097.

Donohue, J.J., Siegelman, P., 1991. The changing nature of employment discrimination legislation. Stanford Law Review 43, 983-1033.

Farber, H.S., Bazerman, M.H., 1986. The general basis of arbitrator behavior: An empirical analysis of conventional and final-offer arbitration. Econometrica 54, 1503-1528.

Frick, B., Malo, M.A., García Martínez, P., Schneider, M., 2012. The demand for individual grievance procedures in Germany and Spain: Labour law changes versus business cycle. Estudios de Economía Aplicada 30, 283-309.

Gabuthy, Y., Lambert, E.-A., 2008. Incitations et licenciement individuel. Revue Française d'Économie 23, 3-50.

Galdón-Sánchez, J.E., Güell, M., 2003. Dismissal conflicts and unemployment. European Economic Review 47, 323-335.

García-Martínez, P., Malo, M.A., 2007. The strategic use of dismissal legislation: An empirical analysis using spanish data. European Journal of Law and Economics 23, 151-167.

Goerg, S.J., Kaiser, J., 2009. Nonparametric testing of distributions-the Epps-Singleton two-sample test using the empirical characteristic function. Stata Journal 9, 454-465.

Goerke, L., Pannenberg, M., 2010. An economic analysis of dismissal legislation: Determinants of severance pay in West Germany. International Review of Law and Economics 30, 71-85.
Ichino, A., Polo, M., Rettore, E., 2003. Are judges biased by labor market conditions? European Economic Review 47, 913-944.

Lazear, E.P., 1990. Job security provisions and employment. Quarterly Journal of Economics 105, 699-726.

Macis, M., 2001. Il mercato del lavoro e la giurisprudenza in materia di licenziamenti (Italia, 1989-1998). Diritto delle Relazioni Industriali 2, 269-284.

Malo, M.A., 2000. A simple model of severance pay determination: The case of individual dismissals in Spain. Labour 14, 269-290.

Malo, M.A., Pérez, J., 2003. Individual dismissals in Europe and the United States: A model on the influence of the legal framework on firing costs. European Journal of Law and Economics 15, 47-63.

Manzanos, C., 2004. Factores sociales y decisiones judiciales. Sociológica, 127-159.

Marinescu, I., 2003, enero. L'application du droit du travail est-elle biaisée par les conditions économiques? Documento presentado en la Conferencia ADRES en Paris.

Marinescu, I., 2011. Are judges sensitive to economic conditions? Evidence from UK employment tribunals. Industrial and Labor Relations Review 64, 673-698.

Mora, J.J., 2006. La dinámica de los despidos en España. Estudios Gerenciales 98, 131-138.

Muñoz Aranguren, A., 2011. La influencia de los sesgos cognitivos en las decisiones jurisdiccionales: el factor humano. Una aproximación. InDret 2, 1-39.

Rachlinski, J.J., 1996. Gains, losses and the psychology of litigation. Southern California Law Review 70, 113-185.

Tversky, A., Kahneman, D., 1974. Judgment under uncertainty: Heuristics and biases. Science 185, 1124-1131.

Viscusi, W.K., 1999. How do judges think about risk? American Law and Economics Association 1, 26-62.

Yoon, A., 2006. Pensions, politics, and judicial tenure: An empirical study of federal judges, 1869-2002. American Law and Economics Review 8, 143-180. 